\title{
MODELACIÓN DINÁMICA DE LOS SÓLIDOS SUSPENDIDOS TOTALES EN EL HUMEDAL JABOQUE, BOGOTÁ (COLOMBIA)
}

\author{
Dynamic modeling of total suspended solids in the wetland Jaboque, \\ Bogotá (Colombia)
}

Palabras clave: humedales, Jaboque, modelación dinámica, simulación, sólidos suspendidos totales.

Key words: wetlands, Jaboque, dynamic modeling, simulation, total suspended solids.

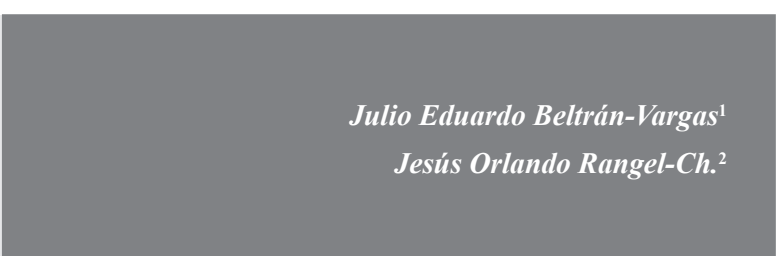

\section{RESUMEN}

Se propone un modelo de simulación dinámico para explicar de manera general el comportamiento de los sólidos suspendidos totales en el humedal Jaboque, Bogotá (Colombia), en tres secciones con características físicas y químicas particulares. El modelo predijo altas concentraciones de sólidos suspendidos totales en abril, mayo, junio, octubre y noviembre, los valores modelados fluctuaron entre $85 \mathrm{mg} \mathrm{L}^{-1}$ y $101 \mathrm{mg} \mathrm{L}^{-1}$ con un promedio de $65.12 \mathrm{mg} \mathrm{L}^{-1}$ con un tiempo hidráulico de retención entre ocho y nueve días año ${ }^{-1}$ para la primera sección, en la segunda sección entre 57 y 69 con un promedio de $50 \mathrm{mg} \mathrm{L}^{-1}$ con un tiempo hidráulico entre 20 y 23 días año ${ }^{-1}$ y en la tercera sección entre $56 \mathrm{mg} \mathrm{L}^{-1}$ y $67 \mathrm{mg} \mathrm{L}^{-1}$ con un promedio de $48,8 \mathrm{mg}$ $\mathrm{L}^{-1}$ y un tiempo hidráulico de retención entre 24 y 26 días año ${ }^{-1}$. En diciembre, enero, febrero, agosto y septiembre se presentó una tendencia a la disminución de los valores modelados. Se compararon los valores estimados de sólidos suspendidos totales con los modelados, de esta forma se evidenció una correspondencia aceptable, $R^{2}=0.95 ; 0.71 ; 0.67$; en todos los casos. El error relativo para cada sección en su orden fue de 0.10 , 0.20 y 0.26 , esto muestra que el modelo no tiende a la sobrestimación de los resultados.

\begin{abstract}
We propose a dynamic simulation model to provide a general explanation for the behavior of total suspended solids in the Jaboque wetland (Bogotá DC). The analyses were performed in three areas with distinct physical and chemical characteristics. The model predicted concentrations of total suspended solids in the months of April, May, June, October and November. Values ranged from $85 \mathrm{mg}$ $\mathrm{L}^{-1}$ and $101 \mathrm{mg} \mathrm{L}^{-1}$ with an average of $65.12 \mathrm{mg} \mathrm{L}^{-1}$ with a hydraulic time retention between eight and nine days per year for the first area, the second area between 57 and 69 with an average of $50 \mathrm{mg} \mathrm{L}^{-1}$ with a hydraulic time between 20 and 23 days per year and in the third area between $56 \mathrm{mg} \mathrm{L}^{-1}$ and 67 $\mathrm{mg} \mathrm{L}^{-1}$ with a mean of $48.8 \mathrm{mg} \mathrm{L}^{-1}$ and a hydraulic retention time between 24 and 26 days per year. The months of December, January, February, August and September showed a tendency to have lower values. The estimated values of total suspended solids based on our model had an acceptable correspondence, $R^{2}=0.95,0.71,0.67$, with the actual values in all cases. The relative error for each area was $0.10,0.20$ and 0.26 , demonstrating that the model does not overestimate the results.
\end{abstract}

\footnotetext{
Universidad Distrital Francisco José de Caldas. Doctorado en Biologiá, Facultad de Ciencias, Universidad Nacional de Colombia. Bogotá, Colombia. jebeltranva@unal.edu.co. Autor para correspondencia

Instituto de Ciencias Naturales, Universidad Nacional de Colombia. Apartado 7495, Bogotá, Colombia. jorangelc@unal.edu.co
} 


\section{INTRODUCCIÓN}

La función natural de los humedales se asemeja al papel de una esponja que recircula el agua en su interior y la libera lentamente, por esto los humedales cumplen funciones esenciales como la regulación de inundaciones y sequías, mantenimiento de la calidad del agua a través de la retención de sedimentos y nutrientes y la remoción de tóxicos. Asimismo, los humedales tienen la capacidad de estabilizar microclimas y retener carbono, que tiene un efecto importante con el calentamiento. Por otro lado, prestan servicios culturales, de educación, recreacion y de turismo (Roldán, 1992; Pinilla, 1996, Wetzel, 2001).

Los humedales han venido sufriendo un proceso de deterioro debido al desarrollo urbano y al mal manejo de las basuras. Como resultado de estas acciones en estos lugares hay un alto grado de contaminación o en el peor de los casos la pérdida total de estos ecosistemas. La acumulación de sólidos suspendidos es uno de los principales problemas de contaminación que presentan los humedales (Prescott \& Tsanis, 1997). Este fenómeno se presenta en el humedal Jaboque pues recibe el impacto de la urbanización y las actividades agrícolas, allí se han encontrado concentraciones se sólidos suspendidos totales (SST) entre 110 y $30 \mathrm{mg} \mathrm{L}^{-1}$ (Álvarez, 2005), con lo cual se incrementan sus condiciones de eutroficación. Por estas razones se propone elaborar la modelación dinámica de los SST, determinar y explicar su relación con el ciclo hidrológico, y predecir de manera general el comportamiento de este material en el humedal.

Los sólidos suspendidos totales (SST) incluyen al plancton, minerales de arcilla, arena, limo, coloides agregados, materia orgánica e inorgánica finamente dividida y otros microorganismos en el agua. Pueden provenir de varias fuentes y se dividen de acuerdo con la composición, ya sea orgánica o inorgánica; pueden originarse en fuentes alóctonas o autóctonas, de levantamiento de tierra o resuspensión (Håkanson, 2004). Los SST regulan dos de las mayores rutas de transporte: la de materiales disueltos en la zona pelágica y la sedimentación de partículas, y la ruta en la zona bentónica (Håkanson et al., 2000; Håkanson et al., 2005). Adicionalmente, son indicadores ambientales para determinar cambios geomorfológicos, contaminación y acciones del cambio climático. Los sedimentos en suspensión transportan cargas de nutrientes, restos de pesticidas, metales pesados y otros elementos. En la cubeta de agua disminuyen la cantidad de luz que penetra, afectan la fotosíntesis y la temperatura del agua; anomalías que están altamente relacionadas con el cambio climático (Salama \& Monbaliu, 2004; Noe \& Harvey, 2007). Los SST también influyen en el transporte de la mayoría de contaminantes (Bradley \& Gilvear, 2000); asimismo, incluyen todos los tipos de material alóctono y autóctono, y se reflejan en la concentración de detritus en la columna de agua. Su variabilidad es alta y muchos factores afectan su comportamiento como la intensidad de la descarga de agua de los tributarios, la intensidad de la precipitación, la temperatura y los vertimientos (Håkanson \& Boulion, 2002; Håkanson et al., 2005; Mora \& Mata, 2007).

\section{MATERIALES Y MÉTODOS}

\section{ÁREA DE ESTUDIO}

El humedal Jaboque se localiza en la localidad de Engativá al occidente de la ciudad de Bogotá, Colombia (Figura 1), está contiguo a la cuenca del río Juan Amarillo, entre el aeropuerto internacional El Dorado y la Autopista Medellín. Limita por el occidente con el río Bogotá, por el sur con los barrios Engativá, Las Mercedes, Puerto Amor, Bolivia, Villa del Mar y la carretera que une a Engativá con el parque La Florida; por el oriente limita con los barrios Álamos Norte, Álamos Sur y Bosques de Mariana, entre otros. Por el norte con los barrios Villas de Granada, Los Ángeles y áreas destinadas al pastoreo y cultivo. Abarca aproximadamente 57 ha y presenta una forma alargada en dirección sur-oriente-noroccidente. En las últimas cuatro décadas, el humedal Jaboque ha visto reducida su extensión por la variación del drenaje, la colmatación por cargas orgánicas de las aguas y vertimientos, ocupación por construcción y fraccionamiento de este. Esta situación ha incidido para que el humedal esté perdiendo su función 
ecosistémica, por una parte, y por la otra, sus atributos como: biodiversidad, estructura, procesos $\mathrm{y}$ funciones.

\section{MÉTODO DE SIMULACIÓN}

El modelo conceptual de los SST se transformó mediante el uso de algoritmos para cada una de las variables. Se desarrollaron las ecuaciones para establecer la relación entre el ciclo hidrológico (precipitación) y la carga de sólidos suspendidos (Anexo 1). La simulación tuvo por objeto conocer y predecir el comportamiento de las fluctuaciones de los SST en sus tres secciones, con relación a la carga de entrada; en especial, al proveniente de los canales Ángeles y Carmelo que recogen las aguas de cuencas aferentes y las vierten en la sección uno. Con el fin de verificar el carácter determinístico y realizar la validación, se realizaron regresiones simples lineares (Legendre \& Legendre, 1998), que permitieron comparar los datos estimados en campo con los resultados modelados y, para evaluar la fortaleza del modelo, se determinó el error relativo (Bryhn \& Håkanson, 2007) y se comprobó el carácter predictivo mediante el análisis de sensibilidad del modelo (Håkanson, 2004; Jorgersen \& Bendoricchio, 2001).

\section{Estructura del modelo de simulación dinamica}

El modelo utilizado para describir la dinámica y el comportamiento de los SST se basa en los principios de balance de masas (Hakanson \& Boulion, 2002; Håkanson 2004) y permite definir al humedal como un tanque reactor; además, se asume la mezcla del humedal como completa durante un intervalo de tiempo (dt). Así, el flujo de materia particulada (SST) o de ingreso de partículas al sistema se puede describir a partir de la ecuación 1 (diferencial).

$V(d c / d t)=Q_{x} C_{i n}-Q_{x} C-M w+R s e d+M s x R r e s$ Ec. 1

donde $V$ es el volumen de la cubeta $\mathrm{m}^{3}, Q$ es el caudal $\left(\mathrm{m}^{3}\right.$ mes $\left.^{-1}\right), M w$ equivale a $\mathrm{C}^{*} \mathrm{~V}$ : Masa húmeda de SST (mg L-1) en la columna de agua, Ms es igual a Mw*Rres: Masa de SST ( $\left.\mathrm{mg} \mathrm{L}^{-1}\right)$ en los sedimentos, Rsed es la tasa de sedimentación, Rres es la tasa de resuspensión, $\mathrm{C}$ en la concentración de SST $\left(\mathrm{mg} \mathrm{L}^{-1}\right)$ y $d c / d t$ representa el cambio de

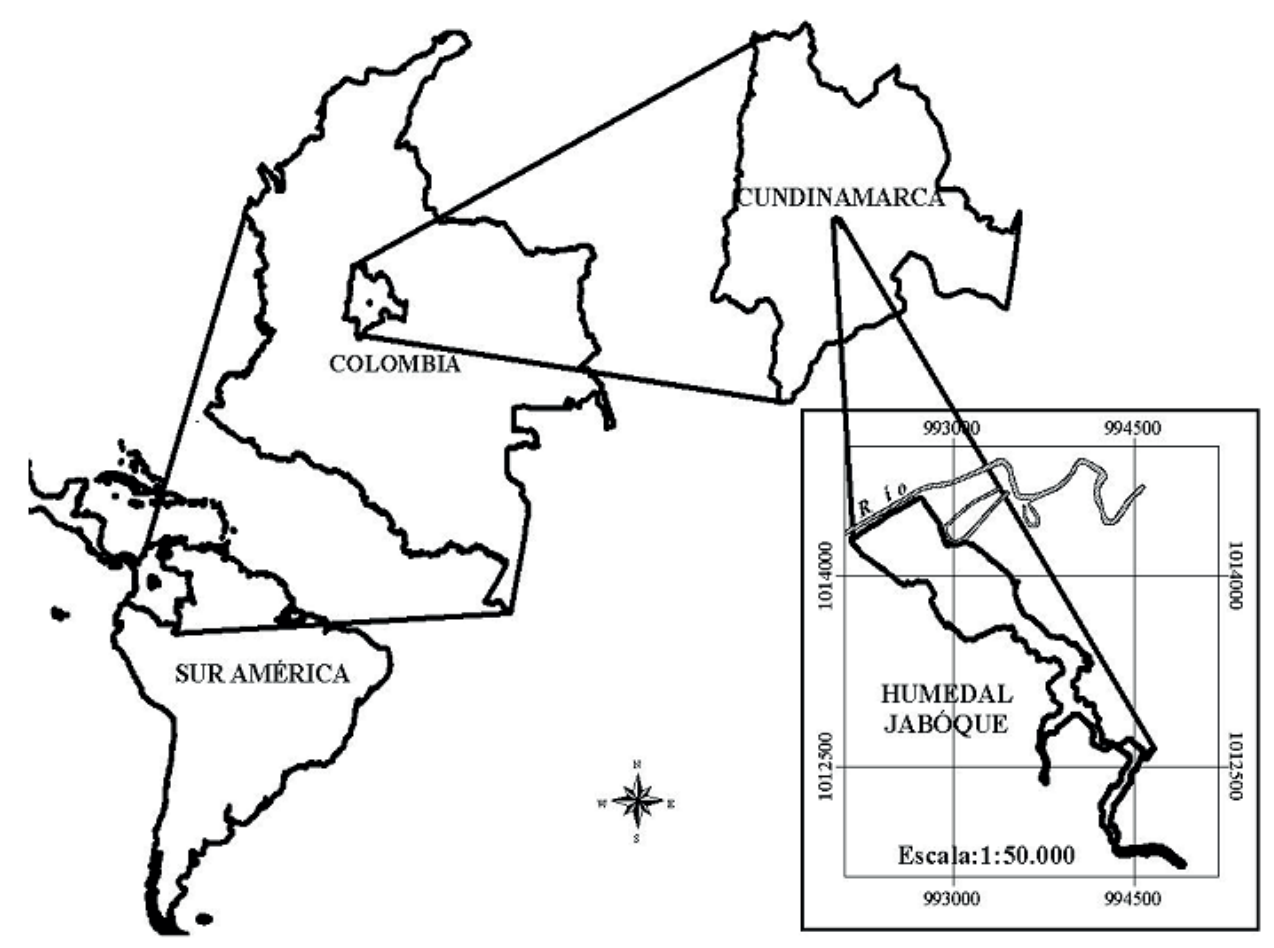

Figura 1. Localización geográfica del humedal Jaboque (Sierra \& Monsalve, 2005) 
concentración (dc) de una substancia por unidad de tiempo, en el tributario o ingreso $Q$ caudal del tributario $\mathrm{m}^{3}$ año ${ }^{-1}$

El modelo tiene en cuenta la descarga de agua del tributario y el flujo de salida en cada cubeta en $\mathrm{m}^{3}$ mes $^{-1}$, la concentración de SST en $\mathrm{mgL}^{-1}$ en cada sección, el área en $\mathrm{m}^{2}$, profundidad en $\mathrm{m}$, volumen de las secciones en $\mathrm{m}^{3}$, la tasa de sedimentación, resuspensión y mineralización (Tabla 1; Håkanson \& Peters, 1995; Håkanson, 2004).

\section{Supuestos del modelo}

El modelo asume los siguientes supuestos, con el fin de delimitar su alcance y posterior generalización o aplicación: en cada una de las secciones del humedal se asume la profundidad media; el caudal de entrada al humedal proviene básicamente de la descarga de la cuenca aferente, y la modelación de la dinámica de los SST se asume, para cada una de las secciones, toda vez que existen barreras morfométricas (estas hacen que cada sección se comporte de manera independiente, pero dependientes de la descarga de la cuenca aferente); el modelo no tiene en cuenta la fracción de SST que se produce por la actividad biológica en el humedal que, si bien es importante, es difícil de calcular con certeza. Debido a que en el humedal Jaboque las diferencias entre temperatura del fondo y superficie son mínimas con tendencia a ser iguales, se asume que no está estratificado y la descarga de la cuenca aferente se calculó con base en la relación existente entre precipitación y área en $\mathrm{km}^{2}$ de la cuenca aferente (Håkanson \& Peters, 1995; Håkanson et al., 2005). Los datos que alimentan el modelo corresponden a un año hidrológico.

\section{RESULTADOS}

La simulación tuvo por objeto conocer y predecir el comportamiento de las fluctuaciones de los SST en cada sección, y determinar su relación con el comportamiento hidrológico del humedal. En la figura 2 y en la tabla 2 se muestran los resultados de la modelación de balance de masas para la sección uno del humedal, allí se observa que el comportamiento de las curvas de precipitación, descarga de la cuenca aferente y la concentración

Tabla 1. Variables del modelo, unidades y fuentes

\begin{tabular}{|c|c|c|}
\hline Variables e índices & Unidades & Fuente \\
\hline $\begin{array}{l}\text { Aporte cuenca aferente (canales Ángeles + } \\
\text { Carmelo ) Flujo de entrada }\end{array}$ & $\mathrm{m}^{3} \mathrm{mes}^{-1}$ & Cálculo a partir de datos del IDEAM. Cálculo \\
\hline Profundidad promedio sección & $\mathrm{m} \mathrm{mes}^{-1}$ & $\begin{array}{l}\text { Datos de campo U. Nacional - Grupo I. Biodiversidad } \\
\text { y Conservación }\end{array}$ \\
\hline Tiempo hidráulico de retención (THR) & días año-1 & Simulación \\
\hline Área sección & $\mathrm{m}^{2} \mathrm{mes}^{-1}$ & $\begin{array}{l}\text { Datos de campo U. Nacional- Grupo I. Biodiversidad } \\
\text { y Conservación Simulación }\end{array}$ \\
\hline Volumen sección & $\mathrm{m}^{3} \mathrm{mes}^{-1}$ & Simulación \\
\hline Carga de entrada de SST & $m g L^{-1}$ & Datos de campo U. Nacional \\
\hline Tasa de sedimentación & mes $^{-1}$ & $\begin{array}{l}\text { Hakanson \& Boulion (2002), Hakanson \& Petres } \\
\text { (1995) }\end{array}$ \\
\hline Tasa de resuspensión & mes $^{-1}$ & $\begin{array}{l}\text { Hakanson \& Boulion (2002), Hakanson \& Petres } \\
\text { (1995) }\end{array}$ \\
\hline Tasa mineralización & mes $^{-1}$ & $\begin{array}{l}\text { Hakanson \& Boulion (2002), Hakanson \& Petres } \\
\text { (1995) }\end{array}$ \\
\hline Masa total SST sección & $\operatorname{gr~m}^{3-1}$ & Simulación \\
\hline Concentración de SST & $\operatorname{gr~m}^{3-1}$ & Simulación \\
\hline Masa de carga interna de SST & $\operatorname{gr~m}^{3-1}$ & Simulación \\
\hline Tiempo hidráulico de retención & días año ${ }^{-1}$ & Simulación \\
\hline Flujo salida sección & $\mathrm{m}^{3} \mathrm{año} 0^{-1}$ & Simulación \\
\hline
\end{tabular}


de los SST son coincidentes con el comportamiento del hidroperiodo. En los meses de enero, febrero, marzo, julio, agosto, que son meses secos en los cuales disminuye la concentración de SST, estos fluctuaron entre $34.9 \mathrm{mg} \mathrm{L}^{-1}$ y $64.8,34.9 \mathrm{mg} \mathrm{L}^{-1} \mathrm{y}$ el tiempo hidráulico de retención (THR) fluctuó entre 11 días año-1 y 24 días año ${ }^{-1}$. Durante abril, mayo, junio, octubre y noviembre se presentan las mayores precipitaciones y descargas de la cuenca aferente, y aumenta la concentración de SST cuyos valores fluctuaron entre 69.2 y $34.9 \mathrm{mg} \mathrm{L}^{-1}-101.7$ y $34.9 \mathrm{mg} \mathrm{L}^{-1}$ y el THR entre ocho días año ${ }^{-1}$ y 14 días año-1. En la tabla 2 se presentan los resultados de la modelación de la precipitación, la descarga de la cuenca aferente, la carga de entrada de SST y modelados, y el THR modelados, de esta manera

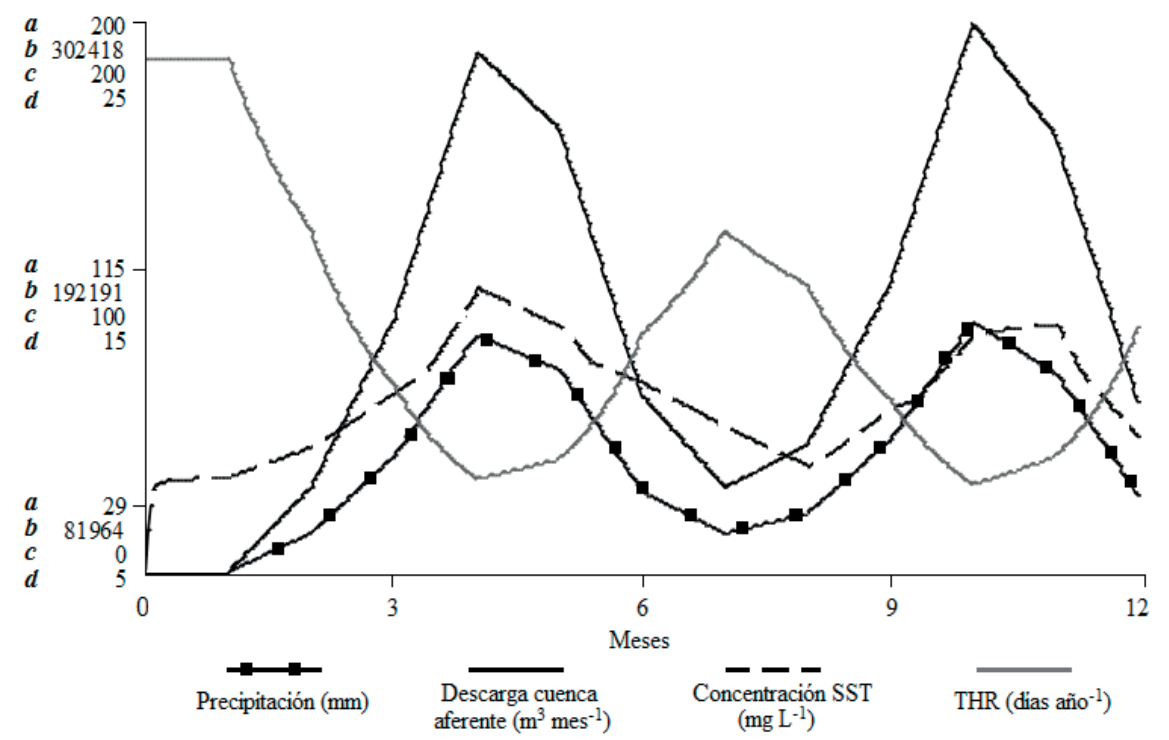

Figura 2. Modelación del balance de masas de SST en el humedal Jaboque sección 1. a. Precipitación $(\mathrm{mm}), \boldsymbol{b}$. Descarga cuenca aferente $\left(\mathrm{m}^{3} \mathrm{mes}^{-1}\right), \boldsymbol{c}$. Concentración SST (mg L-1), $\boldsymbol{d}$. THR (días año $\left.{ }^{-1}\right)$

Tabla 2. Resultados de la modelación del balance de masas de SST

\begin{tabular}{|c|c|c|c|c|c|c|c|c|c|c|c|}
\hline Meses & 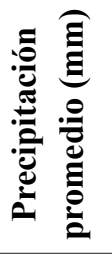 & 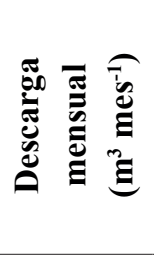 & 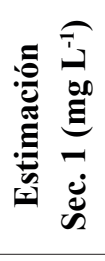 & 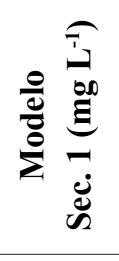 & 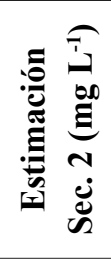 & 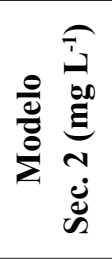 & 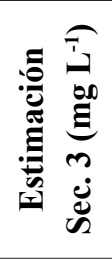 & 孚 & 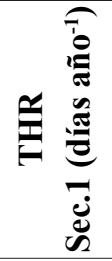 & 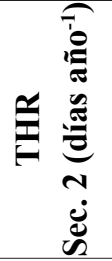 & 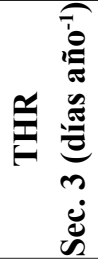 \\
\hline Enero & 29 & 81.964 & 40 & 34.98 & 36 & 18.58 & 30 & 14.4 & 24 & 59 & 68 \\
\hline Febrero & 41 & 115.880 & 50 & 45.3 & 68 & 38.78 & 70 & 36.44 & 17 & 43 & 50 \\
\hline Marzo & 65 & 183.712 & 70 & 64.85 & 83 & 56.68 & 91 & 58.15 & 12 & 29 & 33 \\
\hline Abril & 103 & 291.113 & 91 & 101.76 & 94 & 69.91 & 83 & 60.97 & 8 & 20 & 24 \\
\hline Mayo & 92 & 260.023 & 83 & 89.57 & 74 & 61.95 & 69 & 56.14 & 9 & 22 & 26 \\
\hline Junio & 54 & 152.622 & 69 & 69.23 & 65 & 57.68 & 52 & 47.48 & 14 & 34 & 41 \\
\hline Julio & 41 & 115.880 & 52 & 53.27 & 50 & 48.12 & 38 & 39.19 & 17 & 43 & 50 \\
\hline Agosto & 47 & 132.838 & 38 & 38.88 & 34 & 35.06 & 63 & 46.48 & 15 & 38 & 44 \\
\hline Septiembre & 70 & 197.844 & 63 & 59.03 & 66 & 48.86 & 74 & 53.45 & 11 & 28 & 32 \\
\hline Octubre & 107 & 302.418 & 74 & 85.55 & 75 & 57.71 & 85 & 62.96 & 8 & 20 & 23 \\
\hline Noviembre & 91 & 257.197 & 85 & 89.99 & 81 & 64.73 & 86 & 67.5 & 9 & 23 & 26 \\
\hline Diciembre & 53 & 149.796 & 46 & 49.08 & 47 & 48.09 & 38 & 44.49 & 14 & 34 & 40 \\
\hline Promedio & 66.08 & 186.773 & 63.42 & 65.12 & 64.42 & 50.51 & 64.92 & 48.97 & 13.30 & 32.87 & 38.05 \\
\hline
\end{tabular}


se encontró que estas variables son coincidentes con el comportamiento del hidroperiodo.

En la sección dos, durante los meses de enero, febrero, marzo, julio y agosto, que son meses secos y en los cuales disminuye la concentración de SST, estos fluctuaron entre $18.5 \mathrm{mg} \mathrm{L}^{-1}$ y $56.6 \mathrm{mg} \mathrm{L}^{-1}$ y

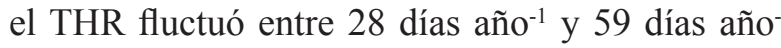
1. En los meses de abril, mayo, junio, octubre y noviembre, los meses con mayores precipitaciones y descargas de la cuenca aferente, aumenta la concentración de SST y los valores fluctuaron entre $69.21 \mathrm{mg} \mathrm{L}^{-1}$ y $57.1 \mathrm{mg} \mathrm{L}^{-1}$ y el THR entre 20 días año ${ }^{-1}$ y 34 días año ${ }^{-1}$. En la sección tres del humedal, en los meses de enero, febrero, marzo, julio y agosto, que son meses secos y en los cuales disminuye la concentración de SST, estos fluctuaron entre $1445 \mathrm{mg} \mathrm{L}^{-1}$ y $58.15 \mathrm{mg}$ $\mathrm{L}^{-1}$ y el THR fluctuó entre 32 días año-1 y 68 días año ${ }^{-1}$. En los meses de abril, mayo, junio, octubre y noviembre, meses con mayores precipitaciones y descargas de la cuenca aferente, tiempo en el que aumenta la concentración de SST, los valores fluctuaron entre $60.9 \mathrm{mg} \mathrm{L}^{-1}$ y $47.8 \mathrm{mg} \mathrm{L}^{-1}$ y el THR entre 20 días año-1 y 24 días año ${ }^{-1}$.

La curva del THR muestra que en la medida que disminuye el THR aumenta la concentración de SST (Figura 2), esto sucede en los meses de mayores precipitaciones y mayores descargas de la cuenca aferente. De esta forma, cuando aumenta el THR, en los meses de menores precipitaciones y menor descarga de la cuenca aferente, disminuye la concentración de SST. En estas condiciones, el tercio alto recibe la mayor cantidad de SST que se transporta desde la cuenca aferente. Lo anterior indica que en épocas de invierno hay mayor transporte de STT, pero su permanencia en las secciones es corta, debido a que los sólidos son lavados por los mayores flujos de agua (Jorgensen, 2003).

Los resultados de la modelación de los SST se muestran en la figura 3 y en la tabla 2 para todas las secciones del humedal. Las curvas indican que los SST se comportan de manera homogénea en las tres secciones e indica la existencia del transporte de sedimentos desde el tercio alto hacia el segundo y tercero. Sin embargo, se hace notorio que los valores de la curva de la sección uno muestran una menor concentración de SST a lo largo del periodo, evento que se puede asociar a una menor capacidad de almacenamiento de esta parte del humedal y, por lo tanto, tiende a funcionar como un canal de paso en condiciones de altas precipitaciones.

\section{SECCIÓN 1. ZONA INTERVENIDA (TERCIO ALTO)}

Esta zona se caracteriza por recibir de manera directa la descarga de SST de origen externo del

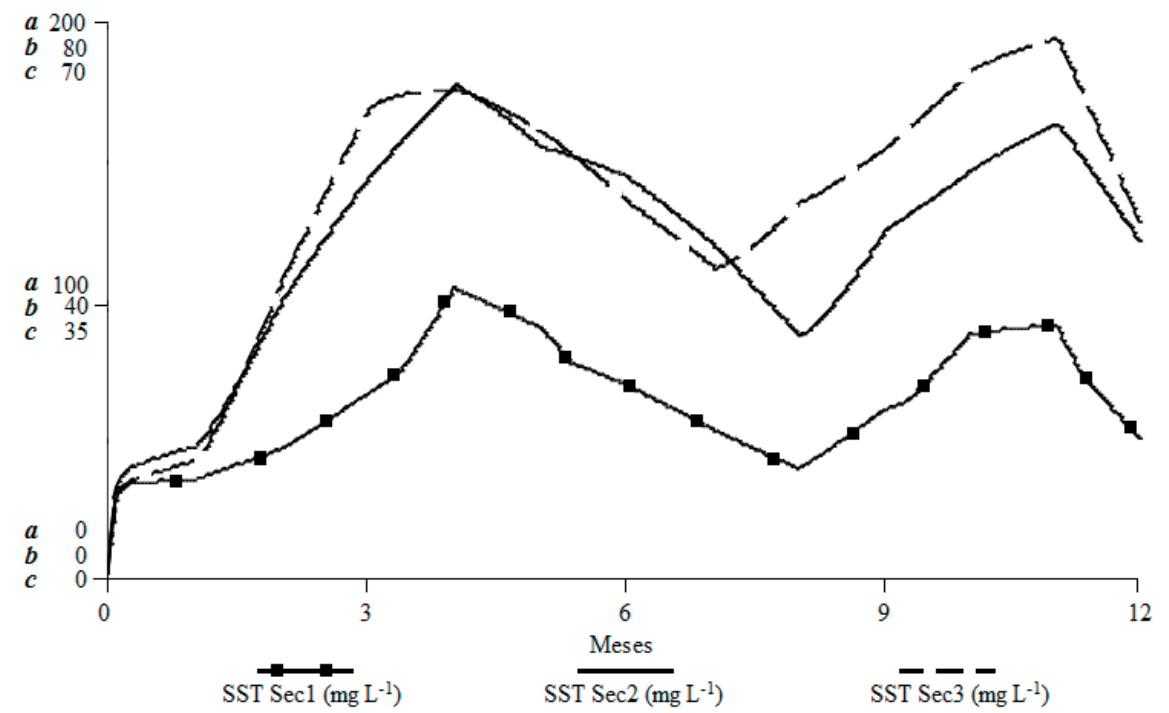

Figura 3. Modelación de los SST en las secciones 1, 2, y 3. $\boldsymbol{a}$. SST Sec1 (mg L-1). b. SST Sec2 (mg L-1), c. SST Sec3 $\left(\mathrm{mg} \mathrm{L}^{-1}\right)$ 
humedal, que corresponde al material particulado que es transportado por el alcantarillado pluvial de la cuenca aferente. En esta cubeta se presentan altas concentraciones de SST, con valores que oscilan entre 38 y $91 \mathrm{mg} \mathrm{L}^{-1}$. Los menores valores corresponden a épocas de verano y los más altos a estaciones lluviosas (Figura 3 y Tabla 2). La comparación entre los valores estimados de SST en campo y los valores modelados arrojó como resultado un buena correspondencia $R^{2}=0.95$, $\mathrm{y}=1.1982 \mathrm{x}+10.862 ;$ los meses con mayores diferencias son enero, marzo, abril, mayo, junio y octubre, en ellos se presentaron diferencias entre los valores estimados y los modelados.

\section{SECCIÓN 2. ZONA DE TRANSICIÓN (TERCIO MEDIO)}

Esta sección, al igual que la primera, se encuentra fuertemente intervenida por la acción antrópica, representada por la descarga de agua que proviene del tercio alto del humedal, ya que parte importante de la misma ha sido desviada hacia los canales perimetrales. En los meses de mayor precipitación se aumentan los SST y en verano disminuyen; adicionalmente, se registran valores desde $34 \mathrm{mg}$ $\mathrm{L}^{-1}$ hasta $94 \mathrm{mg} \mathrm{L}^{-1}$ (Figura 3 y Tabla 2) respectivamente, que indican una fuerte relación con el ciclo hidrológico. La concentración promedio anual fue de $64.2 \mathrm{mg} \mathrm{L}^{-1}$, valor que corresponde a condiciones de eutrofia.

La comparación entre los valores observados y los modelados arrojó una buena correspondencia, $R^{2}=0.7115, \mathrm{y}=06375 \mathrm{x}+9.449$. En este tercio se presentan las mayores concentraciones de SST, aunque estas diferencias no son marcadas, podrían ser parcialmente explicadas por la escorrentía proveniente de actividades agrícolas (Álvarez, 2005), por la remoción de macrófitas por parte de lugareños y por las complejas condiciones morfométricas.

\section{SECCIÓN 3. ZONA CONSERVADA (TERCIO BAJO)}

La principal fuente de SST proviene de la descarga del terciomedio. Se presentan concentraciones entre
$30 \mathrm{mg} \mathrm{L}^{-1}$ y $91 \mathrm{mg} \mathrm{L}^{-1}$ y promedio anual de $64.92 \mathrm{mg}$ $\mathrm{L}^{-1}$. Como en la primera y segunda secciones, el comportamiento de SST está supeditado al ciclo hidrológico. La correspondencia entre los datos observados y modelados fue de $R^{2}=0.6775, y=$ $0.5705 x+11.935$, la cual se considera aceptable, pero las diferencias, al igual que en las anteriores secciones, obedecen a factores morfométricos de la cubeta y biológicos, principalmente. Las diferencias encontradas pueden deberse a que no se han tenido en cuenta los aportes de SST provenientes de actividad biológica del humedal y la descomposición de biomasa del humedal, procesos que aportan una cantidad importante de SST, según Vorosmarty et al. (2003).

\section{ERROR RELATIVO}

Para verificar la capacidad de predicción o fortaleza del modelo se determinó el error relativo (Bryhn \& Håkanson, 2007) para cada una de las secciones. Con este fin se compararon los promedios de los valores de la modelación para cada sección frente a los valores medidos (Tabla 3 ), de acuerdo con las ecuaciones 2 y 3 .

$$
\begin{gathered}
E R=\frac{(\text { valor modelado }- \text { valor medido })}{(\text { valor medido })} \text { Ec. } 2 \\
E R=\frac{(\text { valor modelado sec. } 1-\text { valor medido sec. } 1)}{(\text { valor medido } \text { sección } 1) \text { Ec. } 3}
\end{gathered}
$$

donde $E R$ es el error relativo.

Para un modelo ideal, el valor promedio y la desviación estándar del error relativo deben estar cercanas a un valor de cero $(0)$. Un valor promedio alto positivo significa que las predicciones pueden estar exageradas o sobrestimadas, y cuando los valores son negativos indica que las predicciones pueden estar subestimadas.

Los resultados de la estimación del error relativo de la modelación para la primera sección son de 0.01 y la desviación estándar de 0.09 , esto señala que los resultados de esta no se encuentran sobrestimados. Para la segunda y tercera sección son de -0.20 y -0.23 , con una desviación estándar de 0.16 
y 0.20 , respectivamente; para estas dos secciones los resultados se encuentran entre valores muy cercanos a cero, esto señala la fortaleza del modelo.

\section{ANÁLISIS DE SENSIBILIDAD DEL MODELO}

Para realizar el análisis de sensibilidad del modelo se tuvo en cuenta el promedio anual de precipitación en la zona (p), en la cuenca aferente, como variable independiente, y la concentración SST en cada sección como variable dependiente. Los valores de la precipitación se establecieron según clases entre $400 \mathrm{~mm}^{2}$ ño ${ }^{-1}$ y $1300 \mathrm{~mm}$ año ${ }^{-1}$, a partir del comportamiento histórico en la cuenca aferente, en los últimos 39 años. El análisis de sensibilidad permitió verificar la influencia del ciclo hidrológico en el comportamiento de las concentraciones de SST en las secciones del humedal, también posibilitó confirmar la solidez del modelo en tanto que este refleja la relación existente entre la precipitación, el ciclo hidrológico, la descarga de agua al humedal y la concentración de SST. De esta manera se evidenció que al aumentar la descarga de la cuenca aferente se incrementa la concentración SST en todas las secciones del humedal, pero este fenómeno se manifiesta con una intensidad diferente en cada sección.

\section{Sección 1. Zona intervenida (tercio alto)}

En la figura 4 y en la tabla 4 se aprecian la marcha de la sensibilidad de los SST; el menor valor de concentración se encuentra en la clase de $400 \mathrm{~mm}$ de precipitación y oscila entre 42.2 y $53.4 \mathrm{mg} \mathrm{L}^{-1}$, que corresponde al comportamiento promedio de un año seco. Entre las clases de 800 y $900 \mathrm{~mm}$ de precipitación, la concentración oscila entre 48.7 y $56.2 \mathrm{mg} \mathrm{L}^{-1}$, y a condiciones de altas precipitaciones, entre 1100 y $1300 \mathrm{~mm}$, varía entre 58.3 y $59 \mathrm{mg}$ $\mathrm{L}^{-1}$. Las curvas de concentración muestran un patrón de comportamiento coincidente con el ciclo hidrológico.

Tabla 3. Resultado de la estimación del error relativo del modelo aplicado a todas las secciones. DS. Desviación estándar

\begin{tabular}{lrrr}
\hline Meses & $\begin{array}{c}\text { Error } \\
\text { relativo } \\
\text { Sec. 1 }\end{array}$ & $\begin{array}{c}\text { Error } \\
\text { relativo } \\
\text { Sec. } 2\end{array}$ & $\begin{array}{c}\text { Error } \\
\text { relativo } \\
\text { Sec. 3 }\end{array}$ \\
\hline Enero & -0.13 & -0.48 & -0.52 \\
Febrero & -0.09 & -0.43 & -0.48 \\
Marzo & -0.07 & -0.32 & -0.36 \\
Abril & 0.12 & -0.26 & -0.27 \\
Mayo & 0.08 & -0.16 & -0.19 \\
Junio & 0.00 & -0.11 & -0.09 \\
Julio & 0.02 & -0.04 & 0.03 \\
Agosto & 0.02 & 0.03 & -0.26 \\
Septiembre & -0.06 & -0.26 & -0.28 \\
Octubre & 0.16 & -0.23 & -0.26 \\
Noviembre & 0.06 & -0.20 & -0.22 \\
Diciembre & 0.07 & 0.02 & 0.17 \\
Promedio & $\mathbf{0 . 0 1}$ & $\mathbf{- 0 . 2 0}$ & $\mathbf{- 0 . 2 3}$ \\
DS & $\mathbf{0 . 0 9}$ & $\mathbf{0 . 1 6}$ & $\mathbf{0 . 2 0}$ \\
\hline
\end{tabular}

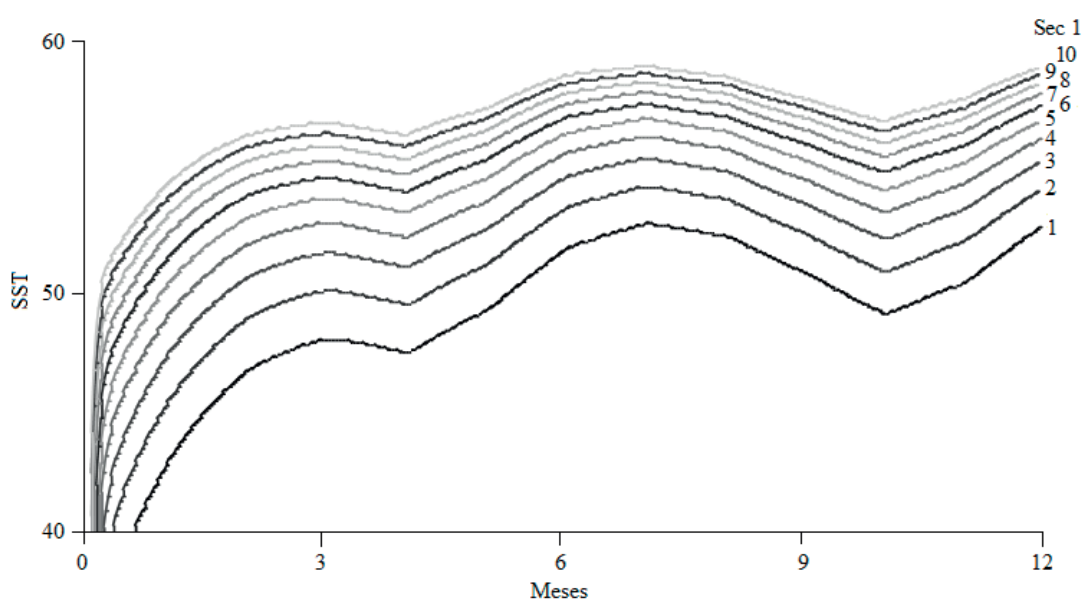

Figura 4. Análisis de sensibilidad del modelo. Variable independiente precipitación $(\mathrm{mm})$, variable dependiente concentración SST $\left(\mathrm{mg} \mathrm{L}^{-1}\right)$ en la sección 1 
Tabla 4. Análisis de sensibilidad del modelo. Variables independientes precipitación, variable dependiente concentración SST en la sección 1

\begin{tabular}{|c|c|c|c|c|c|c|c|c|c|c|}
\hline \multirow{3}{*}{$\begin{array}{c}\text { SERIE } \\
\text { Precipitación }(\mathrm{mm}) \\
\text { Meses } \\
\end{array}$} & 1 & 2 & 3 & 4 & 5 & 6 & 7 & 8 & 9 & 10 \\
\hline & 400 & 500 & 600 & 700 & 800 & 900 & 1000 & 1100 & 1200 & 1300 \\
\hline & \multicolumn{10}{|c|}{ Niveles $\left(\mathrm{mg} \mathrm{L}^{-1}\right)$} \\
\hline Enero & 42.35 & 44.8 & 47.05 & 48.7 & 50.06 & 51.19 & 52.14 & 52.96 & 53.67 & 54.28 \\
\hline Febrero & 46.22 & 48.54 & 50.31 & 51.72 & 52.85 & 53.78 & 54.56 & 55.22 & 55.79 & 56.29 \\
\hline Marzo & 47.67 & 49.8 & 51.43 & 52.7 & 53.73 & 54.58 & 55.29 & 55.89 & 56.41 & 56.86 \\
\hline Abril & 47.18 & 49.28 & 50.89 & 52.16 & 53.2 & 54.05 & 54.77 & 55.39 & 55.92 & 56.38 \\
\hline Mayo & 48.77 & 50.77 & 52.29 & 53.48 & 54.44 & 55.23 & 55.88 & 56.44 & 56.92 & 57.34 \\
\hline Junio & 51.23 & 53.04 & 54.38 & 55.42 & 56.24 & 56.91 & 57.46 & 57.93 & 58.33 & 58.67 \\
\hline Julio & 52.37 & 54.01 & 55.23 & 56.16 & 56.9 & 57.5 & 58 & 58.42 & 58.78 & 59.08 \\
\hline Agosto & 51.96 & 53.57 & 54.78 & 55.71 & 56.46 & 57.07 & 57.58 & 58.01 & 58.38 & 58.7 \\
\hline Septiembre & 50.5 & 52.19 & 53.48 & 54.5 & 55.32 & 55.99 & 56.56 & 57.05 & 57.47 & 57.83 \\
\hline Octubre & 48.83 & 50.65 & 52.06 & 53.17 & 54.09 & 54.84 & 55.48 & 56.03 & 56.51 & 56.92 \\
\hline Noviembre & 49.93 & 51.73 & 53.11 & 54.19 & 55.06 & 55.79 & 56.39 & 56.9 & 57.35 & 57.73 \\
\hline Diciembre & 52.21 & 53.86 & 55.09 & 56.04 & 56.8 & 57.41 & 57.92 & 58.35 & 58.71 & 59.03 \\
\hline Promedio & 49.10 & 51.04 & 52.51 & 53.66 & 54.60 & 55.36 & 56.00 & 56.55 & 57.02 & $\mathbf{5 7 . 4 3}$ \\
\hline
\end{tabular}

Tabla 5. Análisis de sensibilidad del modelo. Variable independiente precipitación, variable dependiente concentración SST en la sección 2

\begin{tabular}{lcccccccccc}
\hline \multirow{2}{*}{$\begin{array}{c}\text { SERIE } \\
\text { Precipitación }(\mathrm{mm}) \\
\text { Meses }\end{array}$} & $\mathbf{1}$ & $\mathbf{2}$ & $\mathbf{3}$ & $\mathbf{4}$ & $\mathbf{5}$ & $\mathbf{6}$ & $\mathbf{7}$ & $\mathbf{8}$ & $\mathbf{9}$ & $\mathbf{1 0}$ \\
\cline { 2 - 11 } & 400 & 500 & 600 & 700 & 800 & 900 & 1000 & 1100 & 1200 & 1300 \\
\hline Enero & 31.75 & 34.78 & 37.32 & 39.47 & 41.31 & 42.91 & 44.31 & 45.54 & 46.63 & 47.61 \\
Febrero & 35.21 & 38.20 & 40.66 & 42.71 & 44.44 & 45.93 & 47.21 & 48.33 & 49.32 & 50.19 \\
Marzo & 35.59 & 38.52 & 40.91 & 42.90 & 44.59 & 46.03 & 47.28 & 48.37 & 49.33 & 50.18 \\
Abril & 32.78 & 35.62 & 37.98 & 39.97 & 41.68 & 43.16 & 44.45 & 45.59 & 46.61 & 47.52 \\
Mayo & 35.76 & 38.69 & 41.09 & 43.08 & 44.78 & 46.22 & 47.48 & 48.57 & 49.54 & 50.40 \\
Junio & 41.67 & 44.59 & 46.90 & 48.78 & 50.32 & 51.61 & 52.69 & 53.62 & 54.43 & 55.13 \\
Julio & 44.30 & 47.04 & 49.18 & 50.88 & 52.26 & 53.40 & 54.36 & 55.17 & 55.86 & 56.47 \\
Agosto & 43.21 & 45.83 & 47.89 & 49.54 & 50.89 & 52.02 & 52.98 & 53.79 & 54.50 & 55.12 \\
Septiembre & 39.41 & 42.02 & 44.12 & 45.84 & 47.29 & 48.51 & 49.57 & 50.49 & 51.30 & 52.02 \\
Octubre & 35.24 & 37.87 & 40.04 & 41.86 & 43.41 & 44.76 & 45.93 & 46.97 & 47.89 & 48.72 \\
Noviembre & 37.93 & 40.69 & 42.92 & 44.78 & 46.34 & 47.68 & 48.84 & 49.84 & 50.73 & 51.52 \\
Diciembre & 44.08 & 46.81 & 48.95 & 50.67 & 52.08 & 53.24 & 54.23 & 55.06 & 55.78 & 56.41 \\
\hline Promedio & $\mathbf{3 8 . 0 8}$ & $\mathbf{4 0 . 8 9}$ & $\mathbf{4 3 . 1 6}$ & $\mathbf{4 5 . 0 4}$ & $\mathbf{4 6 . 6 2}$ & $\mathbf{4 7 . 9 6}$ & $\mathbf{4 9 . 1 1}$ & $\mathbf{5 0 . 1 1}$ & $\mathbf{5 0 . 9 9}$ & $\mathbf{5 1 . 7 7}$ \\
\hline
\end{tabular}

\section{Sección 2. Zona de transición (tercio medio)}

En la figura $5 \mathrm{y}$ en la tabla 5 se aprecia la marcha de sensibilidad de los SST; el menor valor de concentración se encuentra en el nivel $400 \mathrm{~mm}$ de precipitación y oscila entre 31.7 y $44 \mathrm{mg} \mathrm{L}^{-1}$, que corresponde al comportamiento promedio de un año seco. Entre las clases de 800 y $900 \mathrm{~mm}$ de precipitación, la concentración oscila entre 39.7 y $56.2 \mathrm{mg} \mathrm{L}^{-1}$, y en condiciones de alta precipitación, entre las clases 1100 y $130 \mathrm{~mm}$, varía entre 56.4 y $46.1 \mathrm{mg} \mathrm{L}^{-1}$ Similar a la sección anterior, la concentración de los SST mantiene un patrón de comportamiento coincidente con el ciclo hidrológico.

\section{Sección 3. Zona conservada (tercio bajo)}

En la figura 6 y en la tabla 6 se aprecia la marcha de sensibilidad de los SST; el menor valor de concentración se encuentra en la clase de $400 \mathrm{~mm}$ de precipitación y oscila entre 29.6 y $42.2 \mathrm{mg} \mathrm{L}^{-1}$, que corresponde al comportamiento promedio de un año seco. Entre las clases 800 y $900 \mathrm{~mm}$ de precipitación, la concentración oscila entre 37.5 y $50.4 \mathrm{mg} \mathrm{L}^{-1}$, y a condiciones de altas 
precipitaciones, entre las clases 1100 y $1300 \mathrm{~mm}$, varía entre 45 y $55.3 \mathrm{mg} \mathrm{L}^{-1}$. Al igual que la anterior sección, la concentración de los SST mantiene un patrón de comportamiento coincidente con el ciclo hidrológico. Se infiere que para todas las secciones, y aún en condiciones de bajas precipitaciones, las concentraciones de STT se encontrarían entre 44 y $53 \mathrm{mg} \mathrm{L}^{-1}$, esto indica la permanencia de las condiciones de eutrofia, que tiende a incrementarse en la medida que aumenta la descarga de la cuenca aferente. El análisis de sensibilidad del modelo reproduce en parte la relación entre las condiciones hidrológicas y la dinámica de los SST para cada una de las secciones, de allí su importancia en términos predictivos.

\section{DISCUSIÓN}

En condiciones de alta precipitacion, cuando disminuye el tiempo hidráulico de retención, aumenta la concentración de SST que se transportan a través del humedal y esto se depositan en el tercio bajo, donde ocasionan procesos de sedimentación importantes, en la zona más conservada del humedal. En la primera sección, los valores modelados fluctuaron entre $85 \mathrm{mg} \mathrm{L}^{-1}$ y $101 \mathrm{mg} \mathrm{L}^{-1}$ con un promedio de $65.12 \mathrm{mg} \mathrm{L}^{-1}$ con un THR entre ocho y nueve días año ${ }^{-1}$; en la segunda entre 57 y 69 con un promedio de $50 \mathrm{mg} \mathrm{L}^{-1}$ con un THR entre 20 y 23 días año ${ }^{-1}$, y en la tercera sección entre $56 \mathrm{mg}$ $\mathrm{L}^{-1}$ y $67 \mathrm{mg} \mathrm{L}^{-1}$ con un promedio de $48.8 \mathrm{mg} \mathrm{L}^{-1}$ y un THR entre 24 y 26 días año ${ }^{-1}$. En periodos secos,

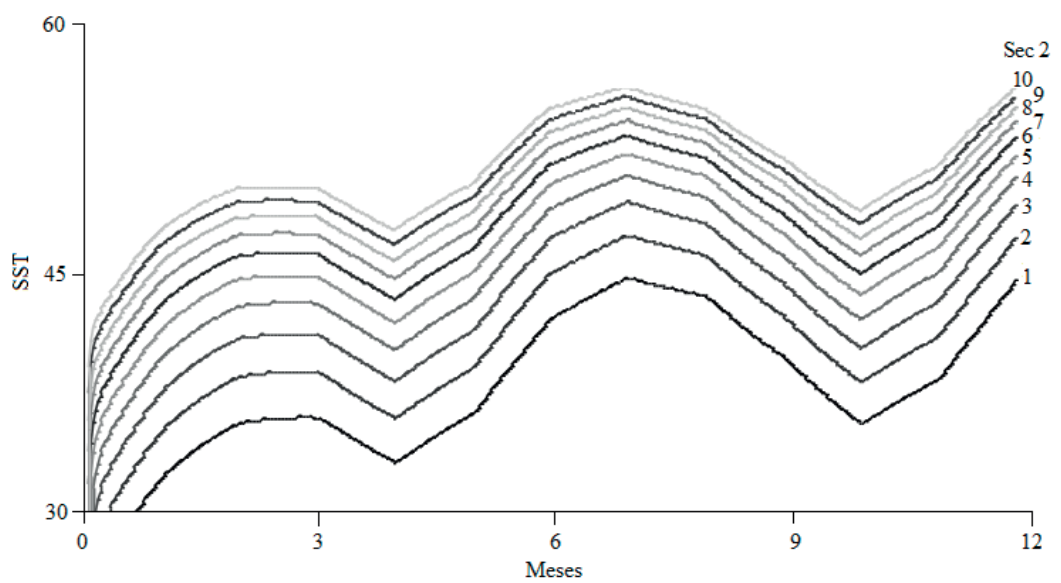

Figura 5. Análisis de sensibilidad del modelo. Variable independiente precipitación $(\mathrm{mm})$, variable dependiente concentración SST $\left(\mathrm{mg} \mathrm{L}^{-1}\right)$ en la sección 2.

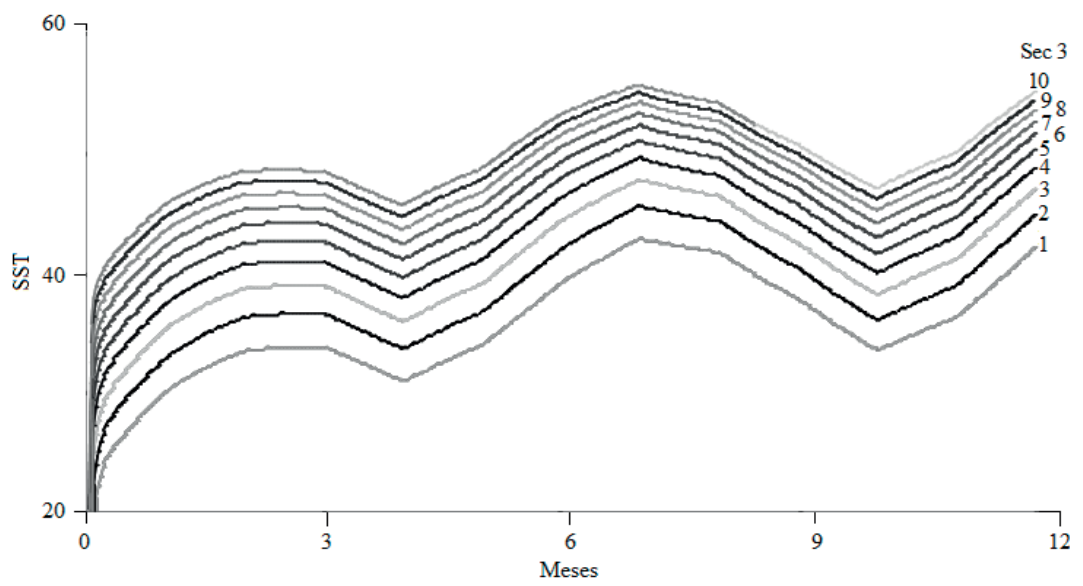

Figura 6. Análisis de sensibilidad del modelo. Variable independiente precipitación $(\mathrm{mm})$, variable dependiente concentración SST $\left(\mathrm{mg} \mathrm{L}^{-1}\right)$ en la sección 3. 
Tabla 6. Análisis de sensibilidad del modelo. Variables independientes precipitación. variable dependiente concentración SST en la sec. 3

\begin{tabular}{|c|c|c|c|c|c|c|c|c|c|c|}
\hline \multirow{3}{*}{$\begin{array}{c}\text { SERIE } \\
\text { Precipitación }(\mathrm{mm}) \\
\text { Meses }\end{array}$} & 1 & 2 & 3 & 4 & 5 & 6 & 7 & 8 & 9 & 10 \\
\hline & 400 & 500 & 600 & 700 & 800 & 900 & 1000 & 1100 & 1200 & 1300 \\
\hline & \multicolumn{10}{|c|}{ Niveles $\left(\mathrm{mg} \mathrm{L}^{-1}\right)$} \\
\hline Enero & 29.67 & 32.71 & 35.30 & 37.51 & 39.43 & 41.11 & 42.58 & 43.89 & 45.06 & 46.11 \\
\hline Febrero & 33.11 & 36.16 & 38.71 & 40.85 & 42.68 & 44.26 & 45.64 & 46.85 & 47.92 & 48.87 \\
\hline Marzo & 33.48 & 36.47 & 38.95 & 41.04 & 42.82 & 44.36 & 45.70 & 46.88 & 47.92 & 48.85 \\
\hline Abril & 30.64 & 33.51 & 35.93 & 37.99 & 39.77 & 41.32 & 42.69 & 43.91 & 44.99 & 45.97 \\
\hline Mayo & 33.58 & 36.58 & 39.07 & 41.17 & 42.96 & 44.50 & 45.85 & 47.04 & 48.08 & 49.02 \\
\hline Junio & 38.77 & 41.83 & 44.31 & 46.35 & 48.05 & 49.49 & 50.71 & 51.77 & 52.69 & 53.50 \\
\hline Julio & 42.55 & 45.50 & 47.82 & 49.70 & 51.23 & 52.51 & 53.59 & 54.50 & 55.29 & 55.97 \\
\hline Agosto & 41.44 & 44.24 & 46.46 & 48.26 & 49.74 & 50.99 & 52.04 & 52.95 & 53.74 & 54.43 \\
\hline Septiembre & 37.54 & 40.27 & 42.49 & 44.32 & 45.87 & 47.19 & 48.33 & 49.33 & 50.21 & 51.00 \\
\hline Octubre & 33.26 & 35.96 & 38.20 & 40.10 & 41.72 & 43.14 & 44.39 & 45.49 & 46.47 & 47.36 \\
\hline Noviembre & 35.91 & 38.76 & 41.09 & 43.05 & 44.72 & 46.15 & 47.39 & 48.48 & 49.45 & 50.31 \\
\hline Diciembre & 41.74 & 44.65 & 46.97 & 48.85 & 50.41 & 51.71 & 52.82 & 53.77 & 54.59 & 55.31 \\
\hline Promedio & 35.97 & 38.89 & 41.28 & 43.27 & 44.95 & 46.39 & 47.64 & 48.74 & 49.70 & 50.56 \\
\hline
\end{tabular}

cuando disminuye la descarga de la cuenca aferente y aumenta el tiempo hidráulico de retención, se reduce la concentración de SST, disminuyendo el transporte entre las secciones del humedal. En la primera sección los valores modelados fluctuaron entre $34 \mathrm{mg} \mathrm{L}^{-1}$ y $59 \mathrm{mg} \mathrm{L}^{-1}$ con un promedio de $65.12 \mathrm{mg} \mathrm{L}^{-1}$ con un THR entre 24 y 15 días año $^{-1}$; en la segunda sección entre $18 \mathrm{mg} \mathrm{L}^{-1}$ y $48 \mathrm{mg} \mathrm{L}^{-1}$ con un promedio de $50 \mathrm{mg} \mathrm{L}^{-1}$ con un THR entre 58 y 38 días año ${ }^{-1}$, y en la tercera sección entre $14 \mathrm{mg}$ $\mathrm{L}^{-1}$ y $46 \mathrm{mg} \mathrm{L}^{-1}$ con un promedio de $48.8 \mathrm{mg} \mathrm{L}^{-1} \mathrm{y}$ un THR entre 68 y 44 días año-1. Rivera (2011) y Mejía (2011) mencionaron que los sólidos suspendidos en el humedal Jaboque disminuyen en las épocas secas y aumentan en la temporada invernal, lo cual confirma la influencia del comportamiento del ciclo hidrológico en este fenómeno.

Los resultados de la modelación dinámica en el humedal Jaboque corroboraron la dependencia entre las concentraciones de SST y el ciclo hidrológico, lo cual sugiere que la concentración de los SST depende más de la descarga del tributario, que de la producción autóctona. Este fenómeno se encuentra influenciado por el tiempo hidráulico de retención, que cuando disminuye aumenta la descarga, permitiendo que se incremente la velocidad de transporte de partículas a través de cada sección. Esto implica que la última sección del sistema, la más conservada, recibe una mayor cantidad de SST, lo cual afecta con mayor intensidad este tercio, condicion que se presenta en épocas de mayores precipitaciones.

En las épocas secas, la tendencia es inversa, el tiempo hidráulico de retención aumenta y las concentraciones de SST disminuyen en todas las secciones del humedal, porque se reduce la descarga de la cuenca aferente. De esta forma se corrobora la influencia del ciclo hidrológico en el comportamiento de los SST; es decir, que en los meses de mayor precipitación se aumentan los SST y en verano disminuyen. Hernández et al. (2008) reseñaron que en épocas de estiaje o verano se dan las más altas concentraciones de sólidos suspendidos, situación contraria a otros estudios, donde se relaciona el fenómeno con las condiciones hidrológicas. Velázquez et al. (2007) en la ciénaga Colombia, Caucasia, Antioquia, y Montoya \& Aguirre (2010), en la Ciénaga de Escobillitas, Córdoba, encontraron que los sólidos suspendidos disminuyen en épocas de verano y aumentan en invierno. Jorgensen (2003) sugiere que a mayores descargas de la cuenca aferente se transporta mayor cantidad de material, pero, a su vez, los tiempos hidráulicos de retención disminuyen, generando un fenómeno de lavado en la cubeta.

El análisis de sensibilidad del modelo reproduce en parte la relación entre las condiciones hidrológicas 
y la dinámica de los SST, para cada una de las secciones, de allí su importancia en términos predictivos. Es de anotar que para todas las secciones y aún en condiciones de bajas precipitaciones (< $500 \mathrm{~mm}$ año $^{-1}$ ), las concentraciones de STT se encontrarían entre 44 y $53 \mathrm{mg} \mathrm{L}^{-1}$, lo cual indica un estado eutrófico para todo el humedal, condición que tiende a incrementarse en la medida en que aumenta la descarga desde la cuenca aferente. Las pruebas para verificar la capacidad de predicción del modelo y su fortaleza y los análisis de sensibilidad del modelo permiten afirmar que la estructura y las variables del modelo reproducen de manera general el comportamiento de los SST del humedal Jaboque. El modelo propuesto, con los debidos ajustes, podria utilizarse para conocer el comportamiento de los SST en otros humedales en el Distrito Capital y del área de influencia del río Bogotá, especialmente.

\section{AGRADECIMIENTOS}

Agradecemos al Grupo de Investigación Biodiversidad y Conservación de la Universidad Nacional de Colombia-Instituto de Ciencias Naturales y a la Empresa de Acueducto y Alcantarillado de Bogotá (EAAB-ESP) por el aporte de la información relacionada con el estudio de caso. A la Rectoría, la Facultad del Medio Ambiente y Recursos Naturales de la Universidad Distrital Francisco José de Caldas por el apoyo económico otorgado para realizar el programa de doctorado. Al Centro de Investigaciones y Desarrollo Científico (CIIDC) y el Grupo de Investigación Indesos, quienes apoyaron la financiación para la adquisición de los programas computacionales, el equipo de computo y la bibliografía especializada que se utilizó en la investigación.

\section{REFERENCIAS BIBLIOGRÁFICAS}

Álvarez, J.P. (2005). Evaluación del estado trófico del humedal Jaboque: Análisis espacial y temporal de las características físico-químicas del agua y de la comunidad plantónica. Bogotá D.C.: Informe final convenio de cooperación Empresa de Acueducto y Alcantarillado de Bogotá - Universidad
Nacional de Colombia - Instituto de Ciencias Naturales. 46 p.

Bradley, C., \& Gilvear, D.J. (2000). Saturated and unsaturated flow dynamics in a floodplain wetland. Hydrological Processes, 14(16), 2945-2958.

Bryhn, A., \& Håkanson, L. (2007). A comparison of predictive Phosphorus load-concentration models for lakes. Ecosystems, 10(7), 1084-1099.

Hakanson, L., \& Peters, H. (1995). Predictive limnology: methods for predictive modelling. SPB Academic publishing. Amsterdam. 456 p.

Hakanson, L., Parparov, A. \& Hambright, K.D. (2000). Modelling the impact of water level fluctuations on water quality (suspended particulate matter) in lake Kinneret, Israel. Ecological Modelling, 128, 101-125.

Hakanson, L., \& Boulion, V. (2002). The lake foodweb: Modelling predation and abiotic/ biotic interactions. Amsterdam. Backhuys Publishers. $344 \mathrm{p}$.

Håkanson, L. (2004). Internal loading: A new solution to an old problem in aquatic sciences Lakes \& Reservoirs. Research and Management, 9(1), 3-23.

Hakanson, L., Mikrenska, M., Petrov, K., \& Foster, I. (2005). Suspended particulate matter (SPM) in rivers: Empirical data and models. Ecological Modelling, 183, 251267.

Hernández, E., Aguirre, N.J., Palacio, J.A., \& Ramírez, J. (2008). Variación espaciotemporal de la asociación fitoplanctónica en diferentes momentos del pulso hidrológico en la ciénaga de Ayapel (Córdoba), Colombia. Actualidades Biológicas, 30(88), 67-81. 
Jorgensen, S.E., \& Bendoricchio, G. (2001). Fundamentals of ecological modelling. Oxford: Elsevier Science Ltda. 530 p.

Jorgensen, S.E. (2003). The application of models to find the relevance of residence time in lake and reservoir management. Science, Management, Education. Limnology, 62, 16-20.

Legendre, P., \& Legendre, L. (1998). Numerical ecology. Amsterdam: Elsevier Science. $853 \mathrm{p}$.

Mejía, D. (2011). Diatomeas perifíticas y algunas características limnológicas de un humedal urbano en la sabana de Bogotá (Tesis de Maestría, Departamento de Biología). Bogotá, D.C.: Universidad Nacional de Colombia. 114 p.

Montoya, Y., \& Aguirre, N. (2010). Dinámica de la producción primaria fitoplanctónica en un lago tropical (ciénaga Escobillitas) a lo largo del pulso de inundación. Revista Facultad de Ingeniería Universidad de Antioquia, 55, 76-89.

Mora, D., \& Mata, A.V. (2007). Aguas para consumo humano y disposición de aguas residuales. San José: Instituto Costarricense de Acueductos y Alcantarillados, Laboratorio Nacional de Aguas. 26 p.

Noe, G.B., \& Harvey, J.W. (2007). Characterization of suspended particles in Everglades wetlands. Limnology and Oceanography, 52(3), 1166-1178.

Pinilla, G. (1996). La problemática de los sistemas acuáticos en Colombia. La Tadeo, 51, 1922.

Prescott, K.L., \& Tsanis, I.K. (1997). Mass balance modelling and wetland restoration. Ecological Engineering, 9, 1-18.
Rivera, J. (2011). Relación entre la composición y biomasa de la comunidad de macroinvertebrados acuáticos y las variables físicas y químicas en el humedal Jaboque Bogotá, Colombia (Tesis de Maestría, Departamento de Biología). Bogotá, D.C.: Universidad Nacional de Colombia. 156 p.

Roldán, G. (1992). Fundamentos de limnología neotropical. Medellín: Universidad de Antioquia. 529 p.

Sierra, O.R. \& Monsalve, C.A. (2005). Zonificación trófica del humedal Jaboque, con base en la familia Bacillariophyceae, Engativá. En J.O. Rangel-Ch. (ed.). Investigación aplicada en restauración ecológica en el humedal de Jaboque (pp. 1 - 23). Acueducto de Bogotá, Universidad Nacional de Colombia.

Salama, S., \& Monbaliu, J. (2004). Quantitative estimation of suspended particulate matter from chris images. Heverlee: ESA Publications Division. 45 p.

Velásquez, J. O., G. Jaime. \& M. Sepúlveda L. (2007). Determinación de la calidad ambiental de la ciénaga Colombia. Caucasia, Antioquia, Colombia. Rev. Gestión y Ambiente, 10 (4), 187-199.

Vorosmarty, C.J., Meybeck, M., Fekete, B., Sharma, K., Green, P., \& Syvitski, J.P. (2003). Anthropogenic sediment retention: Major global impact from registered river impoundments. Global and Planetary Change, 39(1-2), 169-190.

Wetzel, R. (2001). Fundamental processes within natural and constructed wetland ecosystems: Short-term versus long-term objectives. Water, Science and Technology, 44(11-12), 1-8. 
Anexo 1. Ecuaciones del modelo de simulación ecológica humedal Jaboque, componente sólidos suspendidos totales.

\section{Ecuaciones}

M_SST_Sec $1(\mathrm{t})=$ M_SST Sec $1(\mathrm{t}-\mathrm{dt})+(\mathrm{IN}+\mathrm{Car}-$ ga_interna- - Salida-Sedimentación $) * d t$

INIT M_SST_Sec_1 $=25$

INFLOWS:

IN $=($ Promedio_anual_de_Q_año*C_SST_Sec_1)

Carga_interna $=$ Sed_SST_Sec_1*Rres

OUTFLOWS:

Salida $=$ M_SST_Sec_1/TRH_Sec_1

Sedimentación $=$ R_Sed*M_SST_Sec_1

M_SST_Sec_3(t) $=$ M_SST_Sec_3 $(\mathrm{t}-\mathrm{dt})+\left(\mathrm{IN} \_3+\right.$ Carga_interna_3 - Salida_3-Sedimentacion_3 $)^{*} \mathrm{dt}$

INIT M_SST_Sec_3 $=25$

INFLOWS:

IN_3 $=($ Q_Sec_3*CSST_Sec_3 $)$

Carga_interna_3 $=$ Sed_SST_sec_3*Rres_3

OUTFLOWS:

Salida_3 = M_SST_Sec_3/TRH_Sec_3

Sedimentacion_3 $=$ R_Sed_3*M_SST_Sec_3

M_SST_sec_2(t) $=$ M_SST_sec_2(t - dt $)+($ IN_2 + Carga_interna_2 - Salida_2-Sedimentacion_2) $* \mathrm{dt}$

INIT M_SST_sec_2 $=25$

INFLOWS:

IN_2 $=\left(\mathrm{Q} \_\right.$Sec_2*C_SST_Sec_2 $)$

Carga_interna_2 $=$ Sed_SST_Sec_2*Rres_2

OUTFLOWS:

Salida_2 = M_SST_sec_2/TRH_Sec_2
Sedimentacion_2 $=$ R_Sed_2*M_SST_sec_2

Sed_SST_Sec_1 $(t)=$ Sed_SST_Sec_1 $(t-d t)+($ Sedimentación - Carga_interna - MIneralización_sec_1) $* \mathrm{dt}$

INIT Sed_SST_Sec_1 $=10^{\wedge} 4$

INFLOWS:

Sedimentación $=$ R_Sed*M_SST_Sec_1

OUTFLOWS:

Carga_interna $=$ Sed_SST_Sec_1*Rres

MIneralización_sec_1 $=$ Sed_SST_Sec_1*R_min

Sed_SST_Sec_2 $(t)=$ Sed_SST_Sec_2 $(t-d t)+($ Sedimentacion_2 - Carga_interna_2 - Mineralizacion sec_2) $* \mathrm{dt}$

INIT Sed_SST_Sec_2 $=10^{\wedge} 4$

INFLOWS:

Sedimentacion_2 $=$ R_Sed_2*M_SST_sec_2

OUTFLOWS:

Carga_interna_2 $=$ Sed_SST_Sec_2*Rres_2

Mineralizacion_sec_2 $=$ Sed_SST_Sec_2*R_min

Sed_SST_sec_3(t) $=$ Sed_SST_sec_3 $(\mathrm{t}-\mathrm{dt})+($ Sedimentacion_3 - Carga_interna_3 - Mineralización $\sec 3) * \mathrm{dt}$

INIT Sed_SST_sec_3 $=10^{\wedge} 4$

INFLOWS:

Sedimentacion_3 $=$ R_Sed_3*M_SST_Sec_3

OUTFLOWS:

Carga $\_$interna $\_3=$ Sed_SST_sec_3*Rres_3

Mineralización_sec $3=$ Sed_SST_sec_3*R_min

Area_de_cuenca_aferente_2 $=5.7 * 10^{\wedge} 6$ 


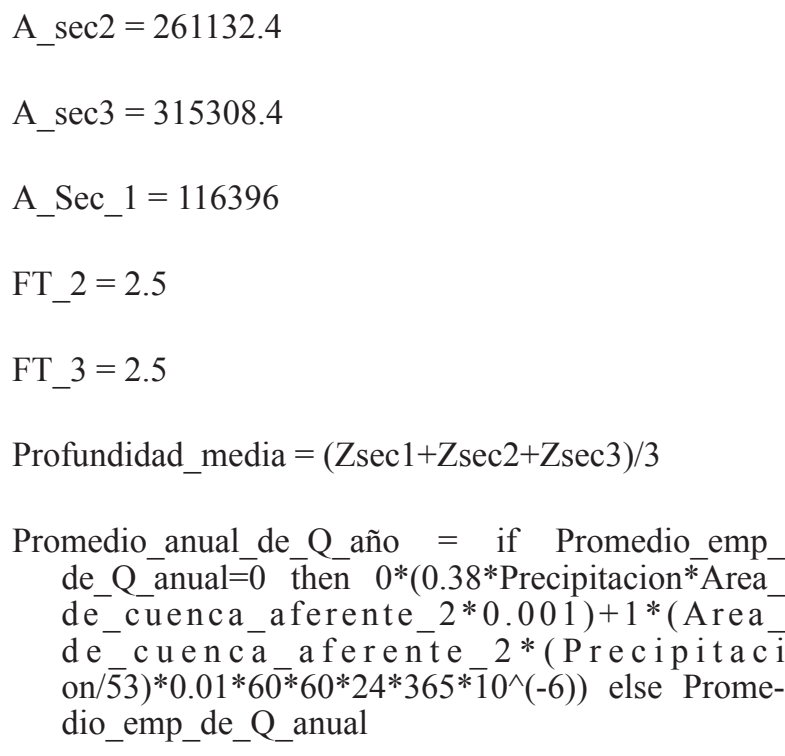

Promedio_emp_de_Q_anual $=0$

Promedio_mensual_de_Q $=$ Promedio_anual_de_Q año/12

P_precipitacion $=840$

Q_Sec_2 $=1.823 \mathrm{e}+006$

Q_Sec_3 $=1.8459 \mathrm{e}+006$

Red_sed $=(72 / 12) * Y S P M$

Red_sed_2 $=(72 / 12)^{*}$ YSPM_2

Red_sed_3 $=(72 / 12) * Y S P M \_3$

Rres $=0.6$

Rres_2 $=0.6$

Rres_3 $=0.6$

R_min $=0.25$

R_Sed $=$ Profundidad_media*Red_sed

R_Sed_2 $=$ FT_2*Profundidad_media*Red_sed_2

R_Sed_3 $=$ FT_3*Profundidad_media*Red_sed_3

SST_Sec_1 $=($ M_SST_Sec_1/Vol_sec1)

SST_Sec_2 $=($ M_SST_sec_2/Vol_sec2 $)$
SST_Sec_3 $=($ M_SST_Sec_3/Vol_sec3 $)$

TRH_Sec_1 $=($ Vol_sec1/Promedio_anual_de_Q_año $)$

TRH_Sec_2 $=($ Vol_sec2/Q_Sec_2 $)$

TRH_Sec_3 $=($ Vol_sec3/Q_Sec_3 $)$

$\mathrm{v}=5$

Volumen_total $=($ Vol_sec1 + Vol_sec2 + Vol_sec3 $)$

Vol_sec1 $=$ A_Sec_1*Zsec1

Vol_sec2 $=$ A_sec $2 * Z s e c 2$

Vol_sec3 $=$ A_sec $3 * Z \sec 3$

$\mathrm{YSPM}=1$

YSPM_2 $=1.0$

YSPM_3 $=1.0$

CSST_Sec_3 = GRAPH(TIME)

$(0.00,0.00),(1.00,50.0),(2.00,70.0),(3.00,91.0)$, $(4.00,83.0),(5.00,69.0),(6.00,52.0),(7.00,38.0)$, $(8.00,63.0),(9.00,74.0),(10.0,85.0),(11.0,46.0)$, $(12.0,38.0)$

C_SST_Sec_2 = GRAPH(MOD(TIME,12))

$(0.00,36.0),(1.00,68.0),(2.00,83.0),(3.00,94.0)$, $(4.00,74.0),(5.00,65.0),(6.00,50.0),(7.00,34.0)$, $(8.00,66.0),(9.00,75.0),(10.0,81.0),(11.0,47.0)$, $(12.0,36.0)$

C_SST_Sec_1 = GRAPH(MOD(TIME,12))

$(0.00,40.0),(1.00,50.0),(2.00,70.0),(3.00,91.0)$, $(4.00,83.0),(5.00,69.0),(6.00,52.0),(7.00,38.0)$, $(8.00,63.0),(9.00,74.0),(10.0,85.0),(11.0,46.0)$, $(12.0,38.0)$

Precipitacion $=$ GRAPH $($ MOD $($ TIME, 24$))$

$(1.00,29.0),(2.00,41.0),(3.00,65.0),(4.00,103)$, $(5.00,92.0),(6.00,54.0),(7.00,41.0),(8.00,47.0)$, $(9.00,70.0),(10.0,107),(11.0,91.0),(12.0,53.0)$, $(13.0,31.0),(14.0,40.0),(15.0,64.0),(16.0,103)$, $(17.0,93.0),(18.0,55.0),(19.0,41.0),(20.0,45.0)$, $(21.0,69.0),(22.0,104),(23.0,90.0),(24.0,51.0)$ 